\title{
Preimplantation genetic diagnosis (PGD) for nonsyndromic deafness by polar body and blastomere biopsy
}

\author{
Gheona Altarescu • Talia Eldar-Geva • Baruch Brooks • Edith Zylber-Haran • \\ Irit Varshaver • Ehud J. Margalioth • Ephrat Levy-Lahad • Paul Renbaum
}

Received: 8 June 2009 /Accepted: 3 August 2009 /Published online: 2 September 2009

(C) Springer Science + Business Media, LLC 2009

\begin{abstract}
Purpose Development of an efficient and reliable PGD protocol for nonsyndromic deafness, by polar body (PB) and blastomere PGD.

Methods The GJB2/GJB6 mutations along with 12 polymorphic markers were used in PGD analysis of blastomeres or polar bodies in 14 couples for 35 cycles. Marker informativity, diagnosis rates, Allele Drop Out (ADO) rates and PB1 heterozygosity rates were assessed.

Results Six cycles were performed by PB biopsy, 27 by blastomere and two combined cycles, resulting in delivery of three unaffected children and five ongoing pregnancies. Diagnosis rates for PB and blastomeres were similar. Only $17 \%$ PB1s were heterozygote. ADO rates of $19 \%$ were observed in both groups.

Conclusions We have developed a single cell multiplex PGD protocol for nonsyndromic deafness with a high efficiency of diagnosis. Most PB1 are homozygous, and similar ADO rates were observed; therefore, blastomere biopsy appears to be the method of choice for this autosomal recessive disease.
\end{abstract}

Keywords Preimplantation genetic diagnosis .

Nonsyndromic deafness · Single cell PCR · Blastomere biopsy · Polar body biopsy · Connexin · GJB2 - GJB6 · PGD · ADO

Capsule A PGD protocol is presented for nonsyndromic deafness. Blastomere analysis was found to be preferable to polar body analysis for the GJB2 and GJB6 genes.

G. Altarescu $(\bowtie) \cdot$ T. Eldar-Geva $\cdot$ B. Brooks $\cdot$ E. Zylber-Haran • I. Varshaver - E. J. Margalioth $\cdot$ E. Levy-Lahad · P. Renbaum Medical Genetics Institute, ZOHAR PGD Lab, and IVF Unit, Shaare Zedek Medical Center, The Hebrew University, Jerusalem, Israel

e-mail: gheona@szmc.org.il

\section{Introduction}

Hearing loss is a significant human disorder. While most deaf individuals can lead normal lives, many are negatively affected by this handicap. In addition, children with cochlear implants show significantly more comorbidity [1]. Hereditary hearing loss occurs in approximately $1 / 2,000$ newborns [2]. More than $50 \%$ of pre-lingual deafness in developed countries is attributed to monogenic defects [3] and of these, $50 \%$ of nonsyndromic recessive cases are caused by mutations in the GJB2 gene (Connexin 26) [4]. The 35delG mutation accounts for a majority of GJB2 mutations detected in the Caucasian population [5] and the 167delT mutation in the GJB2 gene has a high frequency in the Ashkenazi Jewish population [6].

Although nonsyndromic deafness is related to a significant amount of morbidity, studies performed in UK involving genetic screening for deaf participants showed a negative attitude towards genetic testing [7]. In a much larger survey of 644 deaf and 527 hearing subjects with a deaf parent or child, the deaf participants were less likely to agree to genetic testing than the hearing participants [8]. Of the participants that agreed to be tested, a small minority said that they would terminate a pregnancy of a deaf fetus $(2 \%)$. While pregnancy termination may be considered an extreme action taken for a deaf fetus, PGD (transfer of wild type or carrier embryos only) might be the option of choice for this condition.

PGD involves the biopsy and analysis of one or two cells from a $6-8$ cell embryo, or the first and second polar bodies of the oocyte. This technique enables identification of non affected embryos prior to implantation in couples at risk for a Mendelian genetic disorder [9]. Because only unaffected embryos are transferred to the uterus, PGD provides an alternative to current post conception diagnostic procedures 
(amniocentesis or chorionic villous sampling (CVS)), which can be followed by pregnancy termination. The main causes of misdiagnosis in PCR based PGD are the occurrence of allele dropout in single cell analysis and undetected recombination events. Therefore the gold standard for molecular PGD analysis is the use of linked polymorphic markers in combination with mutation specific analysis [10]. These polymorphic markers are selected in the immediate vicinity of the mutation in order to reduce the chances of missing recombination events [11].

Polar body analysis has been shown to be an effective method for maternal autosomal dominant, $\mathrm{X}$ linked and recessive disorders if the couple is willing to accept the possibility of carrier embryos $[10,12,13]$. This method has several advantages: 1) polar body biopsy allows embryo analysis without the removal of any embryonal cells; 2) if the PB analysis is inconclusive a repeat analysis using a single blastomere can be performed at the $6-8$ cell stage; 3 ) $\mathrm{ADO}$ rates have been shown to be significantly less in polar bodies than in blastomeres [13, 14], and 4) informative markers are easier to identify since only the maternal alleles are present. For a majority of genes $60-70 \%$ of first polar bodies are heterozygotes (two alleles) [14]. If two alleles are detected in PB1 the chances of ADO are negligible and the final interpretation of the results depends on analysis of PB2 (if PB2 shows the wild type allele then the oocyte is inferred to be mutant and visa versa). We developed an efficient PGD protocol for use with blastomere and polar body biopsy for nonsyndromic deafness caused by the common mutations in the GJB2 and GJB6 genes. We report the results of six cycles of polar body biopsy and 27 cycles of blastomere biopsy for nonsyndromic deafness over a 4 year period.

\section{Material and methods}

IVF and polar body and blastomere biopsy procedure

IVF treatment was performed by a standard protocol involving pituitary down regulation with GnRH analog, followed by controlled ovarian hyper stimulation with recombinant FSH. For polar body analyis, the first polar bodies (PB1) were removed using the "zona-slitting" technique [14], and Intracytoplasmic sperm injection (ICSI) was then performed on each mature egg. Injected oocytes were transferred to $20 \mu$ l droplets of Global medium (Lifeglobal, Ingenta US) under mineral oil. Approximately $18 \mathrm{~h}$ post injection the oocytes were assessed for fertilization by assessing the presence of two pronuclei ( $2 \mathrm{pn})$, and the second polar body (PB2) was removed using the same procedure as for the first polar body removal. Fertilized oocytes were than placed in fresh $20 \mu \mathrm{l}$ droplets of P1 medium under oil and cultured for a further $48 \mathrm{~h}$. Polar bodies were each carefully transferred to separate $0.5 \mathrm{ml}$ tube containing $5 \mu \mathrm{l}$ of proteinase $\mathrm{K}$ lysis buffer [15]. Blastomere biopsy was performed on day 3 at the $6-10$ cell stage using mechanical "zona-slitting" technique [15]. When performing blastomere analysis each blastomere was separately transferred to a $0.5 \mathrm{ml}$ tube containing $5 \mu \mathrm{l}$ of proteinase K lysis buffer [16]. Analysis was performed on either polar bodies or blastomeres depending on the availability of informative markers and the number of mature oocytes for analysis. When necessary sequential polar body and blastomere analysis was performed. If more than 12 embryos were fertilized, the additional embryos $(2 \mathrm{pn})$ were frozen and analyzed in a separate cycle.

A sample of culture medium (media blank) from each droplet that contained a biopsied polar body was analyzed to verify the absence of maternal cellular genetic material or DNA in the culture medium. In addition, a No Template Control (NTC, reaction blank) was used to monitor absence of external contamination in each PCR reaction.

\section{Molecular analysis}

Genetic testing for the GJB2 35delG and 167delT mutations and the del(GJB6-D13S1830) mutation in addition to microsatellite analysis was performed using fluorescent Polymerase Chain Reaction (PCR) for size discrimination with capillary electrophoresis. DNA was extracted from peripheral blood cells from available family members and CVS samples from previous pregnancies, using a high salt precipitation method [16]. PB1, PB2 and blastomere analysis was carried out following proteinase $\mathrm{K}$ digestion and inactivation at $94 \mathrm{C}$ for $15 \mathrm{~min}$ followed by PCR in a $50 \mu \mathrm{l}$ multiplex PCR reaction containing $0.2 \mu \mathrm{M}$ dNTPs, $10 \% \mathrm{DMSO}, 0.1 \mu \mathrm{M}$ each primer, $1.25 \mathrm{U}$ Taq polymerase in a buffer supplied by the manufacturer (JMR801, UK). The reaction was thermocycled for 30 cycles using a program of $20 \mathrm{~s}$ at $95^{\circ} \mathrm{C}, 1 \mathrm{~min}$. at $62^{\circ} \mathrm{C}$ to $50^{\circ} \mathrm{C}$ and $30 \mathrm{~s}$ at $72^{\circ} \mathrm{C}$. All primer sequences are listed in Table 1. From each multiplex reaction, $1.5 \mu \mathrm{l}$ was used as a template with a hemi-nested primer $5^{\prime}$ fluorescently labeled with 6-FAM, HEX or TAMRA (Metabion, $\mathrm{GmbH}$ ), with either the forward or reverse unlabeled primer for an additional 35 cycle PCR reaction for each individual marker. Reaction products were diluted and run on an ABI Prism 3100 Avant automated sequencer, and analyzed using GeneMapper software (ABI).

Each multiplex protocol included primers for the family specific mutation(s) and 4-8 polymorphic markers flanking GJB2 and GJB6 genes (D13S141, D13S175, D13S633, D13S1275，D13S250，D13S232，D13S1830，D13S292, GJB2-AT1 (chr 13: 19518667), GJB2-AT2 (chr13: 19558847), GJB2-TG1(chr 13: 19622478), GJB2-TG2 
Table 1 Sequence of the primers used in heminested reactions

\begin{tabular}{llll}
\hline Marker/mutation & Forward primer & Reverse primer & Nested primer \\
\hline D13S141 & GGAACGAAGACAATCAGGAG & GTCCTCCCGGCCTAGTCTTA & ACCACGGAGCAAAGAACAGA \\
D13S175 & TATTGGATACTTGAATCTGCTG & CCACAGAAAGTTTTCAGCT & TGCATCACCTCACATAGGTTA \\
D13S633 & ACGCTTTACCCCTAAATC & ACTCGAACTGCTTTGCTTCT & ACGCTTTACCCCTGAAATC \\
D13S1275 & TATTCTGAAGCATCCCAGTG & ATCACTTGAATAAGAAGCCATTTG & TTGTTGCTTCTTACATCATCCT \\
D13S250 & GAAGACAGGGTTATGCCCA & ACAGGATATCCCCAGCAG & CCTGGATGCTAGAGTATTGAAA \\
D13S232 & TTTGAGCCCTTCCTTTCTGA & GGCACAGAAATAAATGTTGAT & TGCTCACTGCTCTTGTGATT \\
D13S1830 & ATTGCTTGAAGCCAGGAGTT & TTTTGTTTGGTACCAGAAGGATTA & CAGCCTGTGCGAGATAGTGAG \\
D13S292 & CGGGGTATTTTAAACAGAAA & TTTGACACTTTCCAAGTTGC & TAATGGCGGACCATGC \\
GJB2-AT1 & CCTGTAGTCCTGGCTCCTT & GTACCTTTGCTTCCCAAAAA & CTGGGGGACACAGTAAGACT \\
GJB2-AT2 & GAGAATGCTGTCAATATTGGTG & ATGGCTGGCATAGAAGAAAA & TCTGCATCACCCAATCAATA \\
GJB2-TG1 & AGATGAAGAGGGAGGCAG & GAAAACAGAACAGAGTTAAAAGGA & ACACCTTCCAAAAACCTAACAC \\
GJB2-TG2 & TTCCTCTCTGTCTCCTTTACTT & GAATCACTTGAACCCAGGAG & TTCTTTGACTCTCCATGCTG \\
GJB2-AC1 & CACAGCTCAGGATTTACCA & CCTTTCTGGTCTAGGCGTC & CCGAAACAGGAGGATTGC \\
GJB2 35 del G & GGTGAGGTTGTGTAAGAGTTGG & AAAATGAAGAGGACGGTGAG & CTCGTGGAGTGTTGTTCCAC \\
GJB2167del T & TTGATCTCCTCGATGTCCTT & GCTCACCGTCCTCTTCATTT & CTTCTTCTCATGTCTCCGGTA \\
\hline
\end{tabular}

(chr 13: 19580216), GJB2-AC1 (chr 13: 19591606)(UCSC, assembly March 2006), [17] (Fig. 1). The amplification, $\mathrm{ADO}$ and contamination rates obtained during preclinical test on unrelated single fibroblasts were within the limits set by the ESHRE PGD consortium guidelines [18], marker exhibiting low amplification rates or high ADO rates were excluded from the PGD assays.

\section{Results}

From March 2004 till January 200914 couples underwent PGD for nonsyndromic deafness at our center. The details of each cycle are presented in Table 2. The mean age of the probands was 30.7 years. In each family a battery of 13 microsatellite markers was tested to identify informative markers for haplotype analysis. Individual PGD strategies based on the familial mutations and informative markers were designed for each family and presented in Table 2. In addition to being carriers of GJB mutations, the proband of couple no. 9 had Neurofibromatosis Type 1. In this couple combined PGD for Neurofibromatosis and connexin was performed using polymorphic markers for both loci. While our marker set allowed us to identify a minimum four fully informative markers flanking the mutations for PB PGD in all families, for blastomere analysis, in no family were we able to identify four markers which could differentiate between all four alleles. However, all families had at least four partially informative markers which could identify one or more normal alleles.

The results of PGD for nonsyndromic deafness are presented in Table 3.
The couples underwent a total of 35 PGD cycles, resulting in a pregnancy rate per embryo transfer of $25 \%$, birth of 3 healthy children (couples 3, 5 and 6), and five ongoing pregnancies. One child born was the result of PB PGD and the other two, blastomere PGD. The mutation status of all three children was re-analyzed after birth (no couples elected to perform CVS or amniocentesis). The blastomere analyses were confirmed with one child wild type and one carrier, the polar body derived pregnancy yielded a carrier with a mutant paternal allele.

Six cycles (17\%) were performed by PB biopsy, 27 cycles $(77 \%)$ by blastomere biopsy and two cycles combined PB and blastomere biopsy. Of the 27 blastomere cycles, 10 were performed with frozen blastomeres. The two cycles that required combined $\mathrm{PB}$ and blastomere biopsy were caused by high number of mutant oocytes (from PB analysis) precluding transfer of more than one embryo without analysis of the paternal allele.

The rate of successful PB diagnosis, 96\% $(n=196)$ was not significantly different $(p=0.08)$ than for blastomere analysis $87 \%(n=157)$ (Table 3$)$. In 19 out of 35 cycles (54\%) two embryos were transferred, in six cycles $(17 \%)$ one embryo was transferred, in seven cycles $(20 \%$,all repeated IVF failure) three embryos were transferred while in three cycle no embryo was available for transfer. The mean number of transferable embryos by $\mathrm{PB}$ analysis was 2.8 and by blastomere analysis 2.6. The mean number of embryos actually transferred for any cycle was 1.9 (1.7 by PB analysis and 1.9 by blastomere analysis). Combined PB and blastomere analysis allowed transfer of two embryos in both of these two cycles. 


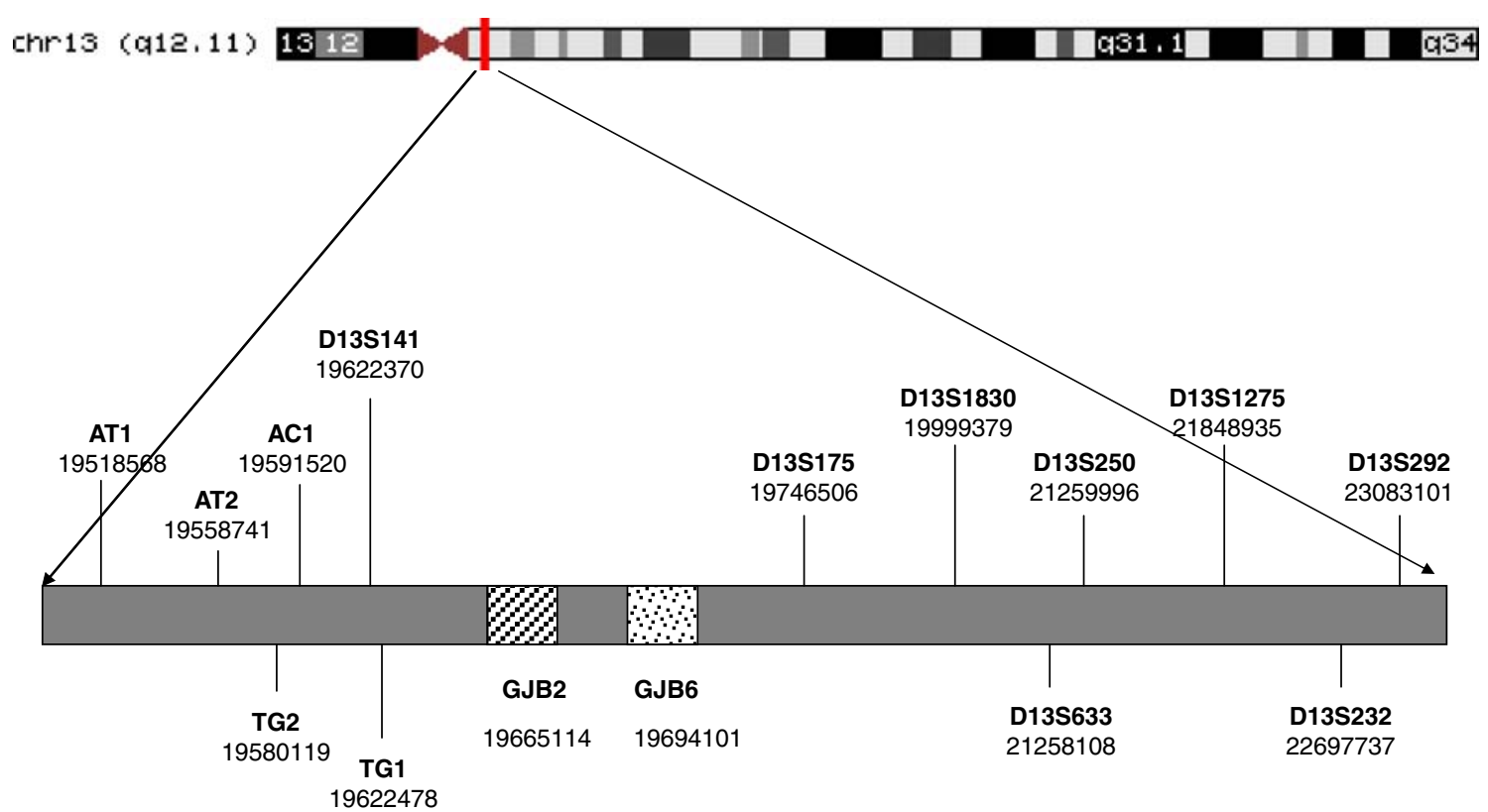

Fig. 1 Schematic representation of polymorphic microsatellite markers on chromosome 13 surrounding the GJB2 and GJB6 genes. The numbering corresponds to the genomic positions of the markers and genes according to March 2006 human reference sequence (NCBI Build 36.1)

The mean number of informative markers available per family was 7.7 for PB analysis, an average of 5.8 were used in each cycle. In blastomere analysis an average of only of 3.3 markers were informative, while an average of another five markers could allow the identification of either the maternal or paternal wild type alleles. A mean of 4.6 informative and partially informative markers were used in the blastomere cycles. The total number of heterozygous PB1s was 19 out of 114PB1 (17\%). No significant difference $(p=0.2)$ was found for ADO rates between heterozygous PB1 reactions 22/135 (16\%), and blastomere reactions 135/687 (19\%). The marker GJB2-AT1 showed the most ADO (24\%) while the marker D13S141 showed the least $\mathrm{ADO}(8 \%)$.

\section{Discussion}

This study describes the development and clinical applications of PGD for nonsyndromic deafness caused by mutations in the GJB2 and GJB6 genes. We have identified a set of 13 polymorphic microsatellite markers flanking these genes, and compared success rates for biopsy, diagnosis, and $\mathrm{ADO}$ in both $\mathrm{PB}$ and blastomere based protocols. Approximately $50 \%$ of autosomal recessive nonsyndromic hearing loss can be attributed to the disorder DFNB1, caused by mutations in the GJB2 and GJB6 genes (encoding the gap junction proteins connexin 26 and 30 ). The carrier rate in the general population for a recessive deafness-causing GJB2 mutation is approximately one in 33 [19].
Differences in the way people view the genetic testing for hearing loss are related to cultural sensitivities in attitudes regarding hearing loss or deafness. The deaf community has traditionally viewed the field of genetics and genetic screening for hearing loss with distrust. In a study of 337 participants (89\% deaf or hard of hearing) $46 \%$ felt that the abortion of a deaf baby should be illegal [20]. Preimplantation genetic diagnosis precludes the need for abortion of affected embryos by analyzing one cell of a 6-8 cell embryo obtained by IVF and transferring to the female unaffected embryos only. While abortion for disabling diseases such as Tay Sachs, Canavan, Fragile X, etc., is viewed as acceptable in most cultures; deaf children have normal intelligence, normal life span, and various surgical interventions are available to allow a normal lifestyle. For these reasons abortion for nonsyndromic deafness is viewed as a non acceptable option for the majority of the population [20]. Moreover, children affected with nonsyndromic deafness using cochlear implants require more hospitalization for postoperative complications such as device extrusion requiring further surgery, wound infection, etc [1]. Taken together, for couples carrying mutations in GJB2 and GJB6 genes, PGD is a preferred option for bearing a non deaf child. All fourteen couples in this study had at least one child diagnosed with nonsyndromic deafness prior to approaching our PGD clinic.

Ever since earlier publications on simultaneous amplification of a mutation specific region with polymorphic markers was shown to improve accuracy of PGD [21] polymorphic markers are used in all PGD analyses. The use 


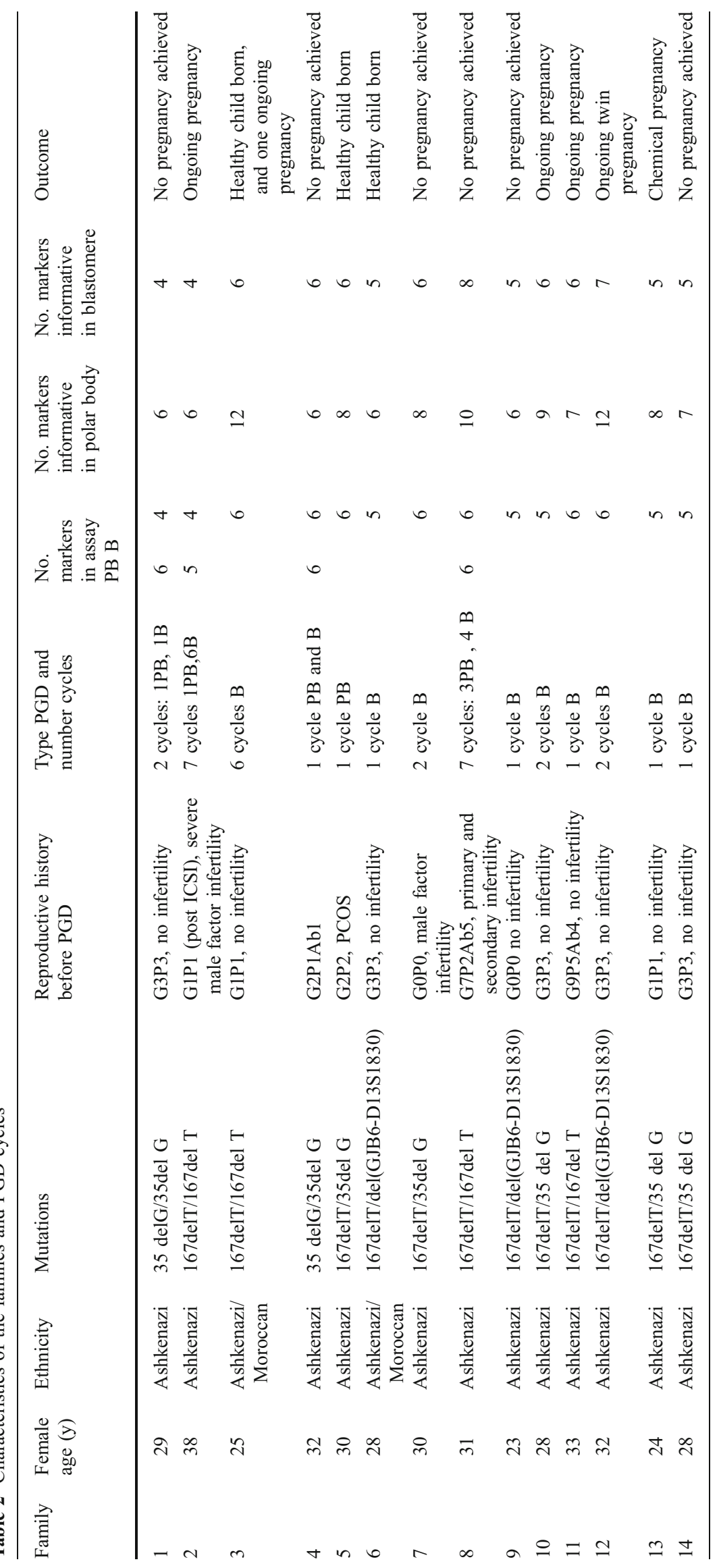


Table 3 Results of PGD for nonsyndromic deafness

\begin{tabular}{ll}
\hline Number families & 14 \\
Total number of markers & 13 \\
Number cycles & 35 \\
Number PB cycles & $6(17 \%)$ \\
Number blastomere cycles & $27(77 \%)$ \\
Number blastomere cycles (frozen) & 10 \\
No. PB cycles continued to blastomere & $2(5 \%)$ \\
Mean number of COC/cycle & 15 \\
Mean number of M2/cycle & 13 \\
Total number of PB1 biopsied & 114 \\
PB1 successfully biopsied & $111(98 \%)$ \\
Total number of PB2 biopsied & 81 \\
PB2 successfully biopsied & $76(93 \%)$ \\
Total embryos diagnosed by PB-PGD & $107(96 \%)$ \\
Total blastomere biopsied & 157 \\
Blastomere successfully biopsied & $153(97 \%)$ \\
Total embryos diagnosed by blastomere-PGD & $133(87 \%)$ \\
Mean embryos transferred/cycle & 1.9 \\
Pregnancy rate/ET & $25 \%$ \\
Number of healthy children born & 3 \\
Number of ongoing pregnancies & 5 \\
Heterozygous PB1 & $19 / 114(17 \%)$ \\
ADO in PB1(total 135 reactions) & $22(16 \%)$ \\
ADO in blastomere (687 reactions) & $135(19 \%)$ \\
Mean number of informative markers/family in PB-PGD & 7.7 \\
Mean number of informative markers/family in blastomere PGD & 3.3 \\
\hline
\end{tabular}

of linked highly polymorphic markers increases the accuracy of diagnosis by reducing misdiagnosis caused by ADO. Informative polymorphic markers (allowing the discrimination between the maternal and paternal alleles) flanking the gene are identified from family genomic DNA samples and used for haplotype construction and the PGD assay. Since PB analysis is based on the discrimination of only the two maternal alleles in the oocyte it is easier to identify informative markers than blastomere analysis which rests on the discrimination of four possible alleles in the embryo. Using our battery of 13 microsatellite linked markers we found an average of 7.7 informative markers for PB analysis as opposed to only 3.3 informative markers available for blastomere PGD. Another factor contributing to this difference may be explained by the fact that most couples at our center were of the same ethnic origin and carried the same founder mutation.

One important advantage of polar body analysis is the heterozygosity in PB1 observed with multiple informative markers and the mutation in usually $60-70 \%$ of cases, therefore excluding ADO and reducing misdiagnosis [14]. In nonsyndromic deafness however, we found that only $17 \%$ of the PB1s were heterozygotes. This is most likely the result of the juxtaposition of the GJB2 and GJB6 genes with the centromere of chromosome 13. Homozygote PB1s do not exclude the possibility of ADO, are more prone for misdiagnosis, and may require blastomere confirmation. While ADO rates have been reported to be lower in PB analysis than blastomere analysis for many disorders [2224], we did not observe any significant difference in ADO rates in our study. The ADO rates we observed in the actual PGD cases were higher than what we observed in individual fibroblasts ( $<10 \%$, data not shown). These rates of ADO can be tolerated when analyzing recessive diseases and using multiple polymorhic markers.

While we originally intended to perform equal numbers of PB and blastomere biopsies, in cases in which a relatively small number of oocytes were retrieved, we performed blastomere biopsy in order that the paternal contribution could improve the outcome of the analysis in this recessive disorder. In addition, the high rate of homozygous PB1s we observed in the initial PB cycles lead us to change our intent and perform blastomere PGD for this disease. Of the 35 cycles we performed, $77 \%$ were blastomere cycles, 17\% were done by PB 1 and 2 analyses, and 2 were done by a combined approach. The rate of successful diagnosis was high and not significantly different between PB and blastomeres (96\% vs 87\%). The overall pregnancy rate was $25 \%$, similar to the pregnancy rates reported by other European centers [25] and resulted in 
three unaffected children, two from blastomere analysis and one from PB PGD, and five ongoing pregnancies.

In conclusion, we have developed a universal single cell multiplex protocol for nonsyndromic deafness with a high efficiency of diagnosis for PGD. Although PB PGD allows more informative marker assessment, most $\mathrm{PB} 1$ are homozygous, and ADO rates were seen to be similar, therefore, blastomere biopsy, appears to be the method of choice for this autosomal recessive disease.

Acknowledgments We would like to thank Rabbi David and Anita Fuld for their generous and ongoing support.

Disclosure of interests Nothing to disclose

Contribution to authorship Gheona Altarescu and Paul Renbaum were the principal investigators and responsible with the design of the methods, genetic counseling and analysis of all cases.

Talia Eldar Geva and Ehud J. Margalioth were the IVF physicians that performed all the IVF cycles of these patients.

Baruch Brooks, Edith Zylber-Haran PhD and Irit Varshaver were the embryologist that performed all the polar body and blastomere biopsies

Ephrat Levy-Lahad was involved in the analysis of the cases.

Ethics approval The PGD is performed in our medical center as routine medical procedure and is not experimental therefore IRB was not required.

Funding The funding was partly provided by the HMOs and partly by a private fund of Fuld family (see acknowledgments).

\section{References}

1. Postelmans JT, Cleffken B, Stokroos RJ. Post-operative complications of cochlear implantation in adults and children: five years' experience in Maastricht. J Laryngol Otol. 2007;121:318-23.

2. Petersen MB, Willems PJ. Non-syndromic, autosomal-recessive deafness. Clin Genet. 2006;69:371-92.

3. Marazita ML, Ploughman LM, Rawlings B, Remington E, Arnos KS, Nance WE. Genetic epidemiological studies of early-onset deafness in the U.S. school-age population. Am J Med Genet. 1993;46:486-91.

4. Rabionet R, Gasparini P, Estivill X. Molecular genetics of hearing impairment due to mutations in gap junction genes encoding beta connexins. Hum Mutat. 2000;16:190-202.

5. Denoyelle F, Weil D, Maw MA, Wilcox SA, Lench NJ, AllenPowell DR, et al. Prelingual deafness: high prevalence of a 30delG mutation in the connexin 26 gene. Hum Mol Genet. 1997;6:2173-7.

6. Morell RJ, Kim HJ, Hood LJ, Goforth L, Friderici K, Fisher R, et al. Mutations in the connexin 26 gene (GJB2) among Ashkenazi Jews with nonsyndromic recessive deafness. $N$ Engl J Med. 1998;339:1500-5.

7. Middleton A, Hewison J, Mueller RF. Attitudes of deaf adults toward genetic testing for hereditary deafness. Am J Hum Genet. 1998;63:1175-80.
8. Middleton A, Hewison J, Mueller R. Prenatal diagnosis for inherited deafness - what is the potential demand? J Genet Couns. 2001;10:121-31.

9. Bick DP, Lau EC. Preimplantation genetic diagnosis. Pediatr Clin North Am. 2006;53:559-77.

10. Verlinsky Y, Cohen J, Munne S, Gianaroli L, Simpson JL, Ferraretti AP, et al. Over a decade of experience with preimplantation genetic diagnosis. Fertil Steril. 2004;82:302-3.

11. Kakourou G, Dhanjal S, Daphnis D, Doshi A, Nuttall S, Gotts S, et al. Preimplantation genetic diagnosis for myotonic dystrophy type 1: detection of crossover between the gene and the linked marker APOC2. Prenat Diagn. 2007;27:111-6.

12. Tomi D, Schultze-Mosgau A, Eckhold J, Schopper B, Al-Hasani $\mathrm{S}$, Steglich C, et al. First pregnancy and life after preimplantation genetic diagnosis by polar body analysis for mucopolysaccharidosis type I. Reprod Biomed Online. 2006;12:215-20.

13. Verlinsky Y, Rechitsky S, Verlinsky O, Kenigsberg D, Moshella J, Ivakhnenko V, et al. Polar body-based preimplantation diagnosis for X-linked disorders. Reprod Biomed Online. 2002;4:38-42.

14. Verlinsky I, Kuliev A. Micromanipulation and biopsy of polar bodies and blastomeres. In: Verlinsky IKA, editor. Atlas of preimplantation genetic diagnosis. 2nd ed. Abrington, Oxon: Taylor and Francis; 2005. p. 15-22.

15. Thornhill AR, McGrath JA, Eady RA, Braude PR, Handyside AH. A comparison of different lysis buffers to assess allele dropout from single cells for preimplantation genetic diagnosis. Prenat Diagn. 2001;21:490-7.

16. Miller SA, Dykes DD, Polesky HF. A simple salting out procedure for extracting DNA from human nucleated cells. Nucleic Acids Res. 1988;16:1215.

17. Karolchik D, Baertsch R, Diekhans M, Furey TS, Hinrichs A, Lu YT, et al. The UCSC genome browser database. Nucleic Acids Res. 2003;31:51-4.

18. Thornhill AR, deDie-Smulders CE, Geraedts JP, Harper JC, Harton GL, Lavery SA, et al. ESHRE PGD consortium 'Best practice guidelines for clinical preimplantation genetic diagnosis (PGD) and preimplantation genetic screening (PGS)'. Hum Reprod. 2005;20:35-48.

19. Estivill X, Fortina P, Surrey S, Rabionet R, Melchionda S, D'Agruma L, et al. Connexin-26 mutations in sporadic and inherited sensorineural deafness. Lancet. 1998;351:394-8.

20. Stern SJ, Arnos KS, Murrelle L, Welch KO, Nance WE, Pandya A. Attitudes of deaf and hard of hearing subjects towards genetic testing and prenatal diagnosis of hearing loss. J Med Genet. 2002;39:449-53.

21. Pickering SJ, McConnell JM, Johnson MH, Braude PR. Use of a polymorphic dinucleotide repeat sequence to detect nonblastomeric contamination of the polymerase chain reaction in biopsy samples for preimplantation diagnosis. Hum Reprod. 1994;9:1539-45.

22. Rechitsky S, Freidine M, Verlinsky Y, Strom CM. Allele dropout in sequential PCR and FISH analysis of single cells (cell recycling). J Assist Reprod Genet. 1996;13:115-24.

23. Rechitsky S, Strom C, Verlinsky O, Amet T, Ivakhnenko V, Kukharenko $\mathrm{V}$, et al. Allele dropout in polar bodies and blastomeres. J Assist Reprod Genet. 1998;15:253-7.

24. Altarescu GE-GT, Brooks B, Margalioth EJ, Lahad A, LevyLahad E, Renbaum P, editor. Polar body versus blastomere: PB or not PB? The American Society of Human Genetics 56th Anual Meeting; 2006; New Orleans, Louisiana.

25. Harper JC, de Die-Smulders C, Goossens V, Harton G, Moutou C, Repping S, et al. ESHRE PGD consortium data collection VII: cycles from January to December 2004 with pregnancy follow-up to October 2005. Hum Reprod. 2008;23:741-55. 